\title{
Ethik und Massenkommunikation bei Papst Paul VI.
}

\author{
von Giselbert Deussen
}

Papst Paul VI. hat nirgends eine systematische Ethik der Massenkommunikation entwickelt. Er nimmt immer konkrete Begegnungen mit den Vertretern von Presse, Sehund Hörfunk zum Anlaß, „um nützlicherweise... darüber nachzudenken, was die Kirche von einem Gegenstand hält, der so wichtig und aktuell ist“", und um zur Entwicklung des Prozesses der sozialen Kommunikation, „über die das kirchliche Lehramt einiges von Vorteil vermitteln kann ${ }^{\alpha}$, Stellung zu nehmen ${ }^{1}$. Da er sich auch in dieser Angelegenheit als höchste kirchliche Lehrautorität begreift - er spricht häufig von einer "Lehre der Kirche" über die „Mittel der sozialen Kommunikation" ${ }^{\text {2 }}$, dürften seine Äußerungen für die kirchliche Publizistik und die Publizistik überhaupr von Gewicht und Bedeutung sein.

Der Papst hat genaue Vorstellungen vom Journalistenberuf, stellt Anforderungen ethischer Art an ihn und zeigt Normen auf, so daß es berechtigt ist, von einer "Ethik der sozialen Kommunikation" bei Papst Paul VI. zu sprechen. Es hat wohl kein Papst zuvor ${ }^{3}$ so häufig und mit soviel Interesse und auch mit soviel Sachverstand über die Medien der Kommunikation gesprochen wie Paul VI. Die Dokumente und Quellen seiner Außerungen sind weit verstreut, von verschiedener Gewichtigkeit und Länge; zu den verschiedensten Anlässen und vor unterschiedlichem Publikum sind sie gemacht.

Seine Außerungen haben formal den Charakter von Grußbotschaften, Briefen, Adhoc-Ansprachen oder programmatischen Reden. Als Medien dienen General- oder Sonderaudienzen, Hốrfunk, Fernseheñ iñu Zeitungen sowie die Verlesung durch Đ̇ auftragte. Anlässe sind vor allem Empfänge von Pressevertretern, Tagungen, Konferenzen, das Konzil, die Errichtung kirchlicher Medieninstitutionen, seine großen Reisen und in der letzten Zeit (seit 1967) der Welttag der sozialen Kommunikation.

\section{Persönliche Verbundenheit des Papstes mit der Presse}

Wie kein Papst vor ihm sucht und schätzt Paul VI. die Gesellschaft der Journalisten. Er ist auch der erste Papst, der mit einem Presseaufgebot auf Reisen ging, zu dem er einen überaus herzlichen Kontakt hatte. Zwei Gründe gibt er selbst an für seine besondere Vorliebe Presseleuten gegenüber: seine eigene Herkunft und Erziehung sein Vater war Journalist ${ }^{4}$ - und sein hohes geistliches Amt, das für ihn - theologisch gesehen - Ahnlichkeiten mit dem Journalistenberuf aufweist und das dem Selbstverständnis des Papstes gemäß gekennzeichnet ist durch den Namen des Völkerapostels Paulus, den er in einer Rede vor Konzilsberichterstattern "einen Vorläufer des Journalistenberufes ${ }^{\alpha}$ nennt: „Wir möchten nur betonen, daß die Hauptbeschäftigung des Völkerapostels, nämlich die Evangelisation der Völker, Uns von nun an am

P. Giselbert Deussen CSSp, Autor des Buches „Die neue liturgische Gemeinde“ (Frankfurt 1968), ergänzt z. Z. seine theologische Ausbildung durch Studien in Publizistik, Soziologie und Christlichen Sozialwissenschaften an der Universität Münster. 
Herzen liegt... Diese Hauptbeschäftigung möge Uns dazu veranlassen, mit grenzenlosem Respekt und mit grenzenloser Bewunderung die Fähigkeit zu betrachten, mit der Sie so reich ausgestattet sind: die Fähigkeit, Nachrichten, Worte, Gedanken und Wahrheit zu verbreiten ${ }^{5} .^{*}$

\section{Der Vorgang sozialer Kommunikationsabläufe in der Sicht des Papstes}

Diese Fähigkeiten und Möglichkeiten zur Missionierung und Einflußnahme durch die Kommunikationsmittel sind es, die den Papst faszinieren. Er schreibt demgemäß den Kommunikatoren eine machtvolle Einflußmöglichkeit auf die Menschen zu; hinter dieser Einschätzung steht eine personalistisch-individualistische Sicht der Kommunikationsprozesse: "Wir betrachten stets mit Ehrfurcht, über welch ein großartiges Instrument, über welch große Macht Sie verfügen ${ }^{6} .^{*}$

Seine Begeisterung läßt ihn die Möglichkeiten besonders der einzelnen Journalisten überschätzen und ihnen eine der Wirklichkeit kaum entsprechende Allmacht in der öffentlichen Meinungsbildung zuschreiben: "Wie sehr sind Wir Uns der Bedeutung bewußt, die Sie in der Welt von heute besitzen mit Ihrer riesigen Gewalt über die öffentliche Meinung7.“ Bemerkungen solcher Art fallen beim Papst sehr häufig und in aufschlußreichen Zusammenhängen.

Paul VI. betrachtet den Kommunikationsablauf via Massenmedien als einen intentional gerichteten Prozeß vom Kommunikator zum Rezipienten; nur so kann man die Bevorzugung und einseitige Beschränkung auf die Rolle des Journalisten bei der Aufstellung von ethischen Normen erklären. Fast alle seine Reden vor Publizisten ließen sich zum Aufweis dieser seiner Sicht des Massenkommunikationsprozesses als „Kommunikation der Einbahnstraße ${ }^{\alpha}$ anführen. Doch dies würde den Rahmen und Raum dieses Beitrags sprengen. Es bleibt jedoch die Begründung dieser Schau interessant, die in einer theologischen Konzeption des Menschen zu sehen ist. Wir wollen sie kurz und in engem Kontakt zu unserem Thema skizzieren.

Die Rede zum zweiten Welttag des Rundfunks ${ }^{8}$ bietet einen Grundriß der Anthropologie Pauls VI., in der die Beziehungen des Individuums zur Gesellschaft durch die "Werkzeuge der sozialen Kommunikation" vermittelt werden.

Im folgenden ist zu beachten, daß der Papst gerade die impersonale, indirekte, technische Vermittlung (hier durch den Hörfunk) nicht anders ausdrüdken kann als durch das Vokabular einer personalistischen Inkarnations- und Schöpfungstheologie, die nur für unmittelbare Relationen von Geist zu Geist (Wort Gottes, personalisiert in Christus) oder von objektiviertem Geist und Geist (Schöpferkraft in der Natur und im Menschengeist) Kategorien kennt: „Es ist die Entdeckung eines göttlichen Gedankens in den geschaffenen Dingen, es ist ein neuer Dialog des Menschen mit dem Kosmos, der auf wunderbare und geheimnisvolle Weise antwortet. Vor allem möchte Unsere Botschaft das Wort grüßen, in dessen Dienst sich der Rundfunk stellt. Das Wort ist Ausdruck des Geistes. Das Radio bietet dem Geist einen Dienst an, den er sich niemals hat träumen lassen, einen Dienst, mit dessen Hilfe er bis zum Himmel dringen kann und bis in die Seele - in einem universalen Ausmaß. Es ist ein Triumph des Geistes. Für Uns, die Wir ,Cultores Verbi', Pfleger des Wortes sind, die Wir das WORT anbeten, das Wort Gottes, das Mensch geworden ist, ist es leicht, im Radio ein sublimes Werk zu sehen, das sich in die Ordnung des Wortes einfügt, das sie zu einer universalen menschlichen Sprache hinführt, ein symbolisches Werk, 
in dem es Uns gefällt, in der natürlichen Ordnung einen Widerschein der erhabenen übernatürlichen Ordnung zu sehen, welche vom Worte Gottes kommt, das sich ausdrückt in schöpferischer Kraft in allen Dingen und das sich in der Geschichte zum Menschen gemacht hat, um unser Meister und unser Heil zu sein in Christus, dem Herrn. ${ }^{\alpha}$

Theologisch relevant und aussageträchtig ist eigentlich nur die personale Kategorie. Das Gegenüber des menschlichen Geistes sind die "Gesetze " und die "Kraft der Natur", über die der Mensch souverän verfügt. Das Eigentliche dieser Verfügung liegt in der "Entdeckung eines göttlichen Gedankens". Die geschaffenen Dinge - hier die Technik - sind nur Durchgangsstadium, kein eigentliches Gegenüber, da sie in das personale Schema nicht passen; ihre Qualität besteht nur darin, „Betätigungsfeld“ des vom Menschen in Treuhänderschaft verwalteten und vertretenen Schöpfergeistes zu sein. Dem entspricht, daß das mediatisierte "Wort" nur "Ausdruck des Geistes" ist, dem das technische Mittel „seinen Dienst anbietet". Dieser Dienst wird - und hier ist die Sicht des Papstes ausgesprochen individualistisch - nur auf den bezogen, der sich des Mittels aktiv bedient.

Alle nun folgenden Bezugslinien sind „Einbahnstraßen“: Menschengeist - Universum, und: Wort - technisches Mittel (wobei Wort aber gleich personalisiert wird als Ausdruck des Geistes). Die Kategorie der Wechselseitigkeit im Sinne eines funktionalen Geschehens fehlt vollständig. Sogar der Begriff „Dialog“, den man doch einer funktionalen Denkfigur eher zuordnen müßte, weist diese „Einbahnstraßen“. Struktur auf. Denn „Dialog “ wird gerade nicht auf das in Interdependenzen sich vollziehende Gespräch der Gesellschaft bezogen, sondern auf das Gegenüber von Menschengeist und Kosmos, ,der auf eine wunderbare und geheimnisvolle Weise antwortet $^{\text {* }}$. Die Realisierung dieser Antwort wird nicht reflektiert: sie ist "wunderbar und geheimnisvoll ${ }^{\alpha}$. Sie soll auch keine Wechselseitigkeit zum Ausdruck bringen, sondern - gemäß der katholischen Naturrechtslehre - das Diktat der "natürlichen Ordnung ${ }^{*}$ sichern, die ja ,ein Widerschein der erhabenen übernatürlichen Ordnung ${ }^{\alpha}$ ist: also wieder eine intentional von oben nach unten gerichtete Linie, die dem Schema der „Einbahnstraße entspricht. Das Verhältnis zwischen beiden Ordnungen wird als "Widerschein“ interpretiert. Dieser Begriff darf nicht als eine Entsprechung der publizistischen Kategorie etwa der „Spiegelung ${ }^{\alpha}$ mißdeutet werden. Hier wird vielmehr theologisch argumentiert, $d$. h. es soll eine Abhängigkeit ausgedrückt werden, die ex definitione nur kausal und einlinig sein kann: die „natürliche Ordnung ${ }^{\alpha}$ als Kopie der ,übernatürlichen ${ }^{\alpha}$, "welche vom Worte Gottes kommt, das sich in schöpferischer Kraft in allen Dingen ausdrückt ${ }^{\alpha}$ - eine spezifisch theologische Denkfigur der „Einbahnstraße ${ }^{\alpha}$, hinter der der Begriff der "Gnade ${ }^{\alpha}$ mit seiner Theologie des „ex gratia misericorditer datum "steht. Hinter und über allem befindet sich eine unveränderliche göttliche Ordnung, die sich „ausdrückt $t^{\alpha}$ im Verhältnis des Menschen zu seiner von ihm geschaffenen oder besser: in der Entdeckung der im Kosmos schon immer vorgegebenen Ordnung. Hier wird an die völlig neuartige Medienkommunikation das alte Grundmuster einer personalen, direkten Kommunikation einer personalisierenden Intimtheologie gelegt, die sich die Vermittlung von (Heils-)Wahrheiten nur in den sprachlichen und logischen Kategorien der „Ich-Du-Beziehung“, der „personalen Begegnung“ oder als ,Akt der personalen Kommunikation ${ }^{\text {" }}$ vorstellen kann, auf denen die ganze Verkündigungstheologie auch organisatorisch als "face-toface ${ }^{\alpha}$-Kommunikation aufgebaut ist. Es überwiegt also die Denkfigur des Statischen, Intentionalen, Hierarchischen, Vorgegebenen, in der naturgemäß dem Kommunikator (Prediger) die beherrschende Rolle zukommt. 
In diesem Sinne ist das Medium immer "nur “ Werkzeug, und zwar ein sehr wirksames, da es den Intentionen des Geistes machtvolle Vervielfältigung gewährt. Diese vom Ordo-Denken geleitete Sicht macht den Kommunikator zum Hierarchen und führt zu einer Mystifizierung des Journalistenberufes, die darin besteht, daß die totale Gnadenhaftigkeit, das „ex gratia datum ${ }^{\alpha}$, d.h. die von Gott einseitig und ohne menschliches Verdienst geschenkte Mittlerschaft Christi, der das Gnadenwort des Vaters an die Menschen ist, vom Papst analog auf den Journalisten als Kommunikator im Verhältnis zu seinen Tätigkeiten im Kommunikationsprozeß angewandt wird, so $\mathrm{da}$ er ihn mit einem Priester vergleichen kann: "Wie der Priester dienen Sie der Sache der Wahrheit... Eine Berufung zum Dienst mit all dem, was dieser einschließt an Opfern, Fruchtbarkeit, Größe und Schönheit".*

Die wesenhafte Funktionalität des dynamischen Hin und Her von Wirkung und Rückwirkung im Kommunikationsprozeß, die Eigendvnamik des Mediums, seine "Zwänge ${ }^{\alpha}$ und „Wirkungen ${ }^{\alpha}$ im sozio-kulturellen Umfeld und die damit gegebene Relativierung der "Macht" des Kommunikators, der selbst nur Teil eines sehr komplexen Gesamtorozesses ist. wird durch solch eine Begrifflichkeit - die für den theologischen Bereich ihre Gïltigkeit haben mag - in keiner Weise abgedeckt und kann demzufolge nicht in ethische Normbegründungen eingehen und darin thematisch werden.

Zusammenfassend muß gesagt werden: im Rahmen der aufgezeigten Denkfiguren und Kateonrien bewoot sich die Kommunikatinnsethik Pauls VI.. d.h. ihre Strukturen sind individualistisch, statisch, naturrechtlich und intentional:

Individualistisch, die ethischen Normen werden auf das Individuum bezogen: der Panst soricht gerne von einer „Deontolooie des Tournalistenherufes ${ }^{\alpha}$, vom ... Berufsethos des Tournalisten ", nie von einem Fthos der Kommunikation als snlther. Es soll damit nicht geleugnet werden, daß dem Papst sehr wohl der gesellschaftliche Bezug dieses Berufsethos vor Augen steht. Es kommt iedoch auf das Aroumentatinnsund Begründungsfundament an: und das ist personalistisch-individualistisch. Dem widersorechen auch nicht die scharfsinnigen Analysen von gesellschaftlichen Wirkungen der Knmmunikationsmittel, da diese stets von einer individualistischen theologischen Anthrooologie her gedeutet werden.

Statisch und naturrechtlich, d. h. die Normfindung selbst geschieht in naturrechtlichen Ableitungen aus dem vorgegebenen Ordo eines als allgemein gültig behaupteten Sittengesetzes.

Intentional, d. h. Garant der Richtigkeit der Normen ist die Kirche als „Hüterin und Lehrerin der Wahrheit ${ }^{\star 10}$, woraus dem katholischen kirchlich gesinnten Tournalisten eine fast unerträgliche Autorität in der Pluralität der Kommunikation als "Gespräch der Gesellschaft ${ }^{\star}$ attestiert wird: "Ihre Tätigkeit kann und muß eine besondere Uberlegenheit gewinnen, welche sich aus der Weisheit ergibt, die nahe am Heiligen Stuhl des Petrus angezündet ist und die Ihre lebendige, treue und moderne Stimme sein will ...11.

Diese Struktur gilt es nun an einem der Begriffe der päpstlichen Kommunikationsethik aufzuzeigen.

\section{Das „Recht auf Information ${ }^{*}$ und seine Begründung in der Sozialnatur des Menschen}

„Es ist ganz klar..., daß das Problem der Information in der Welt von heute sich in einer Weise stellt, die sehr verschieden ist von der, wie sie in den vergangenen 
Jahrhunderten gestellt wurde und dies im Hinblick auf die soziale Evolution. Die Information ist seitdem einmütig als ein universales, unverletzliches und unabdingbares Recht des modernen Menschen anerkannt worden ${ }^{12}$." Die Begründung dieses Rechtes auf Information, so meint der Papst, sehe der "moderne Mensch" in einem „tiefen Bedürfnis seiner sozialen Natur. Um einen Ausdruck zu gebrauchen, den Unser verehrter Vorgänger Johannes XXIII. in seiner Enzyklika ,Pacem in terris ${ }^{c} . .$. gebraucht: ,Jedes menschliche Wesen hat ein Recht auf objektive Benachrichtigung " ${ }^{13}$." Es mag sein, daß der "moderne Mensch" dem Papst in dieser allgemeinen Begründungsformulierung zustimmt. Er darf jedoch keine einhellige Meinung darüber erwarten, was nun inhaltlich mit der "Natur" des Menschen bezeichnet werden kann. An keiner Stelle geht der Papst näher auf dieses Problem ein. Vielmehr argumentiert er von einem formalen Naturbegriff her, den er inhaltlich nicht füllt und als allgemein bekannt und anerkannt vorauszusetzen scheint. $\mathrm{Da}$ die ethische Argumentation immer auf diesen Begriff "Natur" aufsetzt, da dieser auch das Informationsrecht als eine Grundkategorie seiner Informationsethik begründet, bedürfte es einer Klärung dessen, was "soziale Natur des Menschen“ in diesem Zusammenhang bedeutet.

\section{Der Rekurs auf den Naturbegriff}

Was ist mit „Natur", „natürlich“ oder „natürlichen Zwecken gemäß" gemeint? Heißt das in einem ganz allgemeinen und nicht weiter reflektierten Sinn: „vernünftig“ oder "gesund"? "Die Kirche ... wünscht, daß diese Freiheit der Meinung und der Presse ... Gesetze sind, von denen jeder ... einen vernünftigen Gebrauch machen kann. Dieser gesunde Gebrauch der Freiheit, Sie wissen es, ruft nach Erläuterung ${ }^{14}$."

Welcher Naturbegriff der katholischen Naturrechtslehre ist gemeint? Und wie spezifiziert er ethische Normen bezüglich der Massenkommunikation? Ist "Natur" ein biologischer Begriff? Oder ist das metaphysische Wesen des Menschen angesprochen? Wie kann man dann aber erkenntnistheoretisch einwandfrei zur Erstellung einer Ethik an dieses "Wesen" herankommen? Oder ist mit "Natur " eine abstrahierende Vorstellung vom Menschen gemeint, eine Verallgemeinerung konkret erfahrbarer Wirklichkeiten, die der gläubige Mensch im „Schlußverfahren“ von der Offenbarung her als Schöpfungsordnung deutet, die er in seinen Handlungen zu achten und als vorgegebene Gestalt des menschlichen Aktes, als „actus positus“, zu erfüllen hat?: „Sie [die Presse] bemüht sich, die Menschen in einem Klima der Liebe zu erleuchten und $\mathrm{zu}$ unterrichten und sie in die menschliche Gemeinschaft einzufügen, wo die natürlichen Bindungen ihre Verwurzelung in einer gelebten Teilnahme an der großen Familie der Kinder Gottes finden ${ }^{15}$.“

Woher aber erfahren diese "natürlichen Bindungen" ihre allgemein verbindliche Normierung? Denkt der Papst hier an die Aussagen der Pastoralkonstitution, für die sich die „innerste Natur des Menschen" als "Gewissen"16 zeigt, also ein individualethischer Ansatz, der in die Konzeption des Papstes sehr wohl paßte? Diese Frage kann nur durch eine genaue Analyse geklärt werden, für die hier der Platz nicht reicht. Es genügt festzustellen: 1. für den Papst ist der Begriff der „Natur“ des Menschen normierend für eine Ethik der sozialen Kommunikation; 2. die Argumentationsweise mit diesem Begriff ist individualethisch; 3. er scheint diesen Begriff als allgemein bekannt, anerkannt und verpflichtend vorauszusetzen; 4. dieser Begriff gibt seinen kommunikationsethischen Begriffen die entscheidende Einfärbung, so auch dem Begriff des "Rechtes auf Information“. 


\section{Normen bezüglich der Ausübung dieses Rechtes}

Das Recht auf Information ist angesichts der heutigen Weltgesellschaft unveräußerlich für jeden Menschen. Sein Zweck ist das Funktionieren des menschlichen Zusammenlebens. Paul VI. betont immer wieder die Bedeutung dieses Zwecks, ohne jedoch genauer auf das Funktionieren selbst einzugehen. Doch er macht eine wichtige Unterscheidung, die beschreiben soll, wie das Recht auf Information „seinem natürlichen Zweck konform bleibt": „Es ist ein Recht, das zugleich aktiv und passiv ist: das Aufsuchen der Information und die Möglichkeit für alle Menschen, sie zu empfangen ${ }^{17}{ }^{4}$

Es fällt auf, daß Paul VI. differenzierter und genauer als Johannes XXIII. das „universale, unveräußerliche und unverletzliche Recht auf Information" beschreibt. In seiner Enzyklika „Pacem in terris“ (n. 12) erwähnt Johannes lediglich das aktive Recht auf Information im Sinne von "Aufsuchen der Information“, wobei er das passive Recht als prinzipielle Zugänglichkeit nicht benennt. Paul VI. stellt eine Ambiguität dieses einen Rechtes fest, das - immer unteilbar - aktiv und passiv zugleich ist.

Damit umschreibt der Papst sehr gut das, was die Publizistikwissenschaft die „Publizität einer Aussage" nennt und als die grundlegende Kategorie funktionaler Publizistik definiert, auf die alle weiteren Begriffe reduzierbar sein müssen ${ }^{18}$. Man spricht hier von „Publizität" einer Aussage in dem Sinne, daß Aussagemöglichkeit des Kommunikators und Aussagezugänglichkeit für den Rezipienten zwei wesentliche Qualitäten des Aussageprozesses sind ${ }^{19}$.

Der Papst kennt offensichtlich diese Strukturen, ohne sie freilich in ein System zu integrieren, das vor einer einlinigen, intentionalen, kausal-statischen Sicht bewahren würde. Indem er jedoch die aufgezeigten Strukturen auf die Notwendigkeit der Information für das menschliche Zusammenleben bezieht und als ein unveräußerliches Menschenrecht postuliert, bietet er Ansätze eines Moralsystems der Kommunikation, in dem die Begründungseinheit von Sein und Sollen gewahrt ist, d. h. jeder einzelne sittliche Imperativ kann auf allgemeine Seinsgesetze - hier: aktives und passives Recht auf Information als naturrechtliche Grundkategorie - zurückgeführt werden.

Für Paul VI. gründet sich allerdings der Normierungsvorgang nicht im Funktionieren der „Publizität", die als solche schon letzte Norminstanz und Normziel und damit eine vom Ansatz her sozialethische Norm wäre, sondern diese ist für ihn (hier schlägt der individualethische Blickwinkel wieder durch) eine abgeleitete ethische Figur aus der obersten Norminstanz: der "Natur" des Menschen. Der publizistikwissenschaftliche Begriff der "Publizität" ist aber zugleich auch ein empirischer, operationalisierbarer Begriff und deshalb von ganz anderer Qualität als der päpstliche Begriff des aktiven und passiven Informationsrechtes. Dies zeigt sich besonders, wenn der Papst in der Fortsetzung des obigen Zitates ${ }^{20}$ die Relationen zwischen Kommunikator und Rezipienten beschreibt, wobei die Empfängerschaft in eine ausgesprochen passive Rolle abgedrängt wird; sie wird zum Objekt von „Fürsorge“, sie ist zu "schützen“, ihre Rechte sind zu verteidigen, indem der Informator durch Normsetzungen in Schranken gehalten wird; weiter heißt es dann: „Hier erscheint die Wichtigkeit des Informators in ihrem ganzen Licht; er erlaubt jedem durch seine Informationen, die er vermittelt, besser seine Lage zu erkennen und in einer besseren Erkenntnis der Sache seine Entscheidungen zu treffen als ein Mensch, der mit anderen der menschlichen Gruppe, der er angehört, verantwortlich ist. Die Tätigkeit des Informators geht auf diese Weise nicht allein zugunsten des Individuums, sondern auch zugunsten des ganzen Sozialgebildes." 
Die soziale Funktion des „aktiven “ Partners im Informationsprozeß ist hier wieder sehr gut gesehen. Bei aufmerksamem Hinhören ist jedoch zu bemerken, daß diese Funktion ausschließlich als eine sozial-therapeutische beschrieben wird; es fehlt aber vollkommen die sozial-kritische Komponente. Es geht primär darum, der Empfängerschaft „die bessere Erkenntnis einer Sache ${ }^{\text {“ }}$ zu erleichtern, um sich danach in das Sozialgebilde einzufügen. $\mathrm{DaB}$ dies oft mit Kampf der Interessen und Machtkontrolle, mit aggressiver Kritik verbunden sein muß, klingt nicht an. Die aktive, rückkoppelnde Empfängerrelation - eine eminent ethische Größe - ist in bezug auf den Kommunikationsprozeß selbst vollkommen unterschlagen; bezüglich der aktiven Teilnahme am öffentlichen politischen Leben wird sie lediglich auf die Ebene der personalen Einzelentscheidungen des vor-öffentlichen Privatlebens geschoben, die dann bezeichnenderweise nicht auf das "ganze Sozialgebilde“ bezogen ist (diese Relation bleibt vielmehr allein den Kommunikatoren vorbehalten), sondern auf die Vereinzelung „der anderen menschlichen Gruppen", der man sangehört". Welche Rolle dabei das alte Stände-(Ordo-)denken spielt, kann hier nicht untersucht werden; aber es sei erwähnt, daß eine statische Sicht des Gesprächs der Gesellschaft mit einer ständischen Gesellschaftslehre korrespondiert. Die Verantwortung für das „ganze Sozialgebilde" fällt - in Kompetenzabstufungen dosiert - dann nicht mehr in die "unterste" Schicht der Empfängerschaften, sondern in die Zuständigkeit des nächst "höheren" Standes der Informatoren, die "Diener" und "Mittler" $z$ wischen dem Ganzen und seinen Teilen, zwischen "Oben" und „Unten" sind. Der Fortgang der Argumentation des Papstes in dieser Rede ${ }^{21}$ bestätigt diesen Eindruck und leitet zugleich über auf einen Schlüsselbegriff der Informationsethik Pauls VI.: die „Ehrfurcht/Ehrenhaftigkeit" als der „sicherste Garant" einer „gesunden“ Handhabung des Rechtes auf Information.

\section{Die „Ehrfurcht/Ehrenhaftigkeit“ als Regulativ des Informationsrechtes}

Mit diesem Begriff haben wir ein Specificum päpstlicher Normgebungen vor uns, welches dem Papst ein besonderes Anliegen ist, da er es in vielen seiner Reden immer wieder mit Eindringlichkeit und Originalität entfaltet.

Das Recht auf Information - aktiv: als Aufsuchen und passiv: als Empfangsmöglichkeit - findet damit in einem ethischen Prinzip eine Grenze, oder besser: ein Regulativ, das nicht selbst Qualität dieses Rechtsprinzips ist, sondern dem Rechtssubjekt anhaftet und von ihm her dann das Prinzip selbst "färbt", bestimmt und ausrichtet. Dieses Regulativ übersetzten wir mit „Ehrfurcht“. Dieses Wort ist nun keineswegs eindeutig. Hinzu kommt, daß der Papst sich in verschiedenen Sprachen ausdrückt, so daß wir mehrere Begriffsreihen vor uns haben, die an Präzision und Schärfe zu wünschen übrig lassen, aber stets auf die gleiche Grundintention ethischer Regulierung des Informationsrechtes zielen. Die Sinnskala dieser Worte schillert zwischen den Bedeutungen der deutschen, spezifisch moralisierenden Begriffe: „Ehrfurcht " und „Respekt" auf der einen Seite und „Ehrbarkeit" und "Ehrenhaftigkeit" auf der anderen Seite; beide Begriffsreihen finden wir im Italienischen, der Muttersprache des Papstes, in "rispetto “22 und „onesto “23; im Englischen in „respect" ${ }^{\text {“24 und }}$ "honest"; im Französischen in „respectueuse “25 und „respect" 26 und andererseits „honnête ${ }^{\text {"27 }}$ und "noble ${ }^{28}$; im Spanischen in "respeto" 29 . Die Unschärfe dieser Begriffe kommt nicht von ungefähr: in ihnen leuchtet eine breite Skala menschlicher positiv ethischer Verhaltenseinstellungen auf, die sich nur im Kontext konkreten Handelns präzisieren lassen. 
Die erste Begriffsreihe im Sinne von ${ }_{\text {R Respekt/Scheu/Ehrfurcht }}{ }^{\star}$ bezieht der Papst meist auf die Freibeit der Information und will damit die Grenze des Rechtes auf freien Zugang zur Information zum Wohle der Wahrung der Intimsphäre und Würde

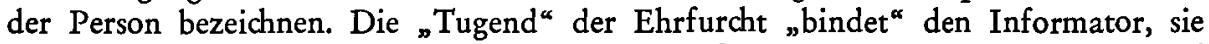
schränkt seine Freiheit ein: „Die Information muß z. B. das Recht der anderen auf ihren guten Ruf respektieren und muß haltmachen vor dem legitimen Geheimnis ihres Privatlebens. Wie viele Verstöße gibt es heute auf diesen beiden Gebieten!... Ehrfurchtsvoll vor dem anderen und seinem Wohlergehen muß die Information sein und mehr noch vielleicht: ebrfurchtsvoll vor dem gemeinsamen Wohl30.“

Leider wird nicht näher ausgeführt, was „ehrfurchtsvoll vor dem gemeinsamen Wohl“ bezeichnet. Das ist insofern bedauerlich, als man hieraus ein Recht ableiten könnte, wodurch kategorisch die Rücksichtnahme aus Staatsraison sanktioniert würde: eine gefährliche Schlußfolgerung, die hier dem Papst sicher fernliegt. Er wird wohl ganz allgemein den gegenseitigen menschlichen Respekt im öffentlichen Leben meinen: „Wir wünschen, daß Ihnen allen immer am Herzen liegt, Ihre freie Tätigkeit zum Dienst am gemeinsamen Wohl in gegenseitigem Respekt auszuüben ... ${ }^{31}$.“

Die zweite Begriffsreihe im Sinne von "Ehrenhaftigkeit/Ehrbarkeit" finden wir im Kontext mit der Forderung nach Wabrhaftigkeit. Sie hat hier nicht abgrenzenden, sondern integrierenden Charakter. Die "Ehrenhaftigkeit/Ehrlichkeit ${ }^{*}$ wird hier vielleicht im Sinne von Camus' "honnêteté $e^{\text {- }}$ als eine "Tugendmaxime " verstanden, die die Wahrhaftigkeit des Informators bezüglich des Informationsrechtes „steuert" "färbt ${ }^{\text {", }}$, vertieft und freisetzt; in diesem Sinne spricht der Papst dann den Journalisten eine „noble mission sociale" ${ }^{\text {"32 }} \mathrm{zu}$ : „Die Pflicht, die Wabrbeit zu suchen, ist bisweilen sehr beschwerlich. Doch der ernste, ebrbare ... Journalist . . erfüllt sie gern....33."

\section{Zusammenfassende Thesen zur päpstlichen Informationsethik:}

1. Der Papst schreibt den "Mitteln der sozialen Kommunikation “ eine entscheidende Rolle für das Zusammen- und Überleben in der modernen Gesellschaft zu.

2. Diese Mittel stellen eine riesige Macht dar im Hinblick auf den Fortschritt oder aber die Gefährdung der Menschheit.

3. Die Basis des "gesunden Gebrauchs" dieser Mittel liegt in einem sittlichen Prinzip, das aus der - wie auch immer zu definierenden - „Natur" des Menschen abzuleiten ist.

4. Die wichtigste Aufgabe der sozialen Kommunikation ist die Information zur Formierung einer auf das Wohl des Einzelnen wie der Gruppen ausgerichteten Gesellschaft.

5. Daraus ist eine Informationsethik abzuleiten.

6. Das Recht auf Information als naturrechtliche Grundkategorie dieser Informationsethik wird definiert als aktives Recht des Zugangs zur Information und als passives Recht auf Unterrichtung.

Die folgenden Thesen gehen über das Dargelegte hinaus, seien aber der Vollständigkeit halber hinzugefügt:

7. Daraus resultiert die Forderung nach Freiheit der Information.

8. Die Freiheit der Information ist nicht Hemmungslosigkeit; ihr regulierendes Prinzip ist die Wahrheit (verbunden mit dem Begriff „Respekt/Ehrenhaftigkeit $\left.{ }^{\alpha}\right)$. 
9. Freiheit und Wahrheit sind dem Wohl des Individuums und der Gemeinschaft dienend einzuordnen.

10. Der Informationsprozeß selbst wird individualethisch normiert (vgl. das Bild der "Einbahnstraße ${ }^{\alpha}$ ).

11. Die Sanktion ist außerhalb des Informationsprozesses selbst zu suchen (Staat bzw. Kirche als Kontrolleur?).

\section{Anmerkungen:}

Wir bedienen uns folgender Abkürzungen:
AAS Acta Apostolicae Sedis.
CS Communicatio Socialis. Zeitschrift für Publizistik in Kirche und Welt.

NS (EI) N.C.W.C. (= National Catholic Welfare Conference, eine Gemeinschaftsorganisation des US-Episkopates; heute: United States Catholic Conference) News Service (Editorial Information).

OR L'Osservatore Romano.

1. Ansprache vor Konzilsberichterstattern vom 29.6.1963, in: OR, 103:1963 vom 3. 7. 1963, S. 3; NS (EI):12. 7. 1963.

2. Brief des Kardinalstaatssekretärs A. G. Cicognani an die Vierte Vollversammlung der katholischen Presse-Union Italiens (Unione Cattolica della Stampa Italiana - U.C.S.I.) 1968 in Tarent mit Anregungen und Wünschen Papst Pauls VI. Veröffentlicht in OR, $108: 1968$ vom 8.11. 1968, S. 1; deutsch in: CS 2:1969, S. 152-157.

3. Vgl. Pius XII. in: A.-F. Utz/ J.-F. Groner: Soziale Summe Pius' XII. Freiburg 1954, nn. $1054-1105,3462,4031,4284-4295,5264-5360,5929-5935$. Vgl. Werner Hamerski: Reden Papst Pius' XII. zu Fragen der Publizistik, in: „Publizistik“, Bremen, 8. Jg. 1964, S. 611-631. Der chronologische Uberblick (S. 627-631) verzeichnet zwar 58 laufende Nummern, die sich aber auf einen Zeitraum von 1942 bis 1958 beziehen. Bei einer Zusammenstellung der Reden Pauls VI. zu diesem Thema verzeichnen wir seit 1963 immerhin 54 publizistisch relevante Äußerungen.

4. Siehe unter 1; ferner: Audienzansprache an Künstler und Publizisten vom 6. 5.1967, in: AAS LIX:1967, S. 505-509; deutsch: CS 1:1968, S. 143-148.

5. Radiobotschaft anläßlich der Zweiten Weltwoche des Hörfunks ("Settima mondiale della Radio") am 8.11.1964, in: AAS LVI:1964, S. 1020.

6. Audienzansprache vor der katholischen Presse-Union Italiens (U. C. S. I.), am 22. 9. 1963, in: OR, 103:1963 vom 23./24.9.1963; ferner: NS (EI):13.12.1963.

7. Ansprache vor Konzilsberichterstattern vom 1. 10. 1963, in: AAS LV:1963, S. 864-866; deutsch vgl. „Herderkorrespondenz", Freiburg i. Br., 18:1963/64, S. 153 f. - Vgl. Audienzansprache an den 15. Kongreß der "Presse périodique “ in Rom vom 30.4.1965, in: AAS LVII:1965, S. 507-510. - Vgl. ferner: Ansprache an Journalisten auf der Rüdkreise vom Besuch der UNO in New York nach Rom, gehalten am 4.10. 1965 auf dem Kennedy-Airport/New York, in: AAS LVII:1965, S. 891 f. Deutsch: „KNA-Konzilssonderdienst" " Nr. 58, vom 6.10.1965.

8. Siehe unter 5 .

9. Ansprache an die päpstliche Kommission für die publizistischen Mittel vom 28.9.1964, in: AAS LVI:1964, S. 939-941.

10. Wir beziehen uns hier auf das Dekret über die publizistischen Mittel Nr. 3, 13 und 17.

11. Siehe unter 9.

12. Audienzansprache an die Tagungsteilnehmer des "Séminaire des Nations Unies sur la liberté de l'information" in Rom vom 17.4.1964, in: AAS LVI:1964, S. 387-389.

13. Ebenda.

14. Audienzansprache an den 15. Kongreß der „Presse périodique“ in Rom, siehe unter 7.

15. Brief an Raimondo Manzini, den damaligen Präsidenten der „Union Internationale de la Presse Catholique" = UIPC (heute: Union Catholique Internationale de la Presse $=$ UCIP) rom 7.5.1965, in: AAS LVII: 1965 , S. $579 \mathrm{f}$.

16. Pastoralkonstitution über die Kirche in der Welt von heute, Artikel 16. In: „Konzilsdekrete 4“, Recklinghausen 21966, S. 23.

17. Siehe unter 12.

18. Vgl. F. W. Dröge: Publizistik und Vorurteil, Münster 1967, S. 90. 
19. Vgl. H. Prakke u. a.: Kommunikation der Gesellschaft, Münster 1968, S. 118 f.

20. Siehe unter 12.

21. Ebenda.

22. Hörfunk- und Fernsehansprache zum ersten Welttag der Kommunikationsmittel am 2. 5. 1967, in: AAS LIX:1967, S. 517-520; deutsch in: CS 1:1968, S. 53-57.

23. Siehe unter 2.

24. Ansprache an Journalisten auf der Rüdkreise vom Besuch der UNO in New York, siehe unter 7.

25. Siehe unter 12.

26. Ansprache bei der Audienz für den Rat der "Union Catholique Internationale de la Presse“ (UCIP) am 23.11.1968, in: OR, 108:1968 vom 24.11.1968, deutsch in: CS 2:1969, S. 158-161. Ferner: Siehe unter 14 und Rede vor Konzilsberichterstattern im Konzilspresseamt am 26.11.1965, in: AAS LVII:1965, S. 991-995; deutsch in: „Herderkorrespondenz ${ }^{\star}$, Freiburg i. Br. 20:1966, S. 53.

27. Siehe unter 12 .

28. Ebenda.

29. Rede vor Journalisten auf der Reise zum Eucharistischen Weltkongreß in Bogota am 23. 8. 1968, in: AAS LX:1968, S. $625 \mathrm{f}$.

30. Hörfunkbotschaft anläßlich der Einweihung von "Radio Loyola“ (Bolivien) am 31.7. 1965, in: AAS LVII:1965, S. 728 f.

31. Siehe unter 14.

32. Siehe unter 12.

33. Siehe unter 2; vgl. unter 29.

\section{SUMMARY}

In not one of his speeches Pope Paul VI has yet given systematic ethics for mass communications. He always uses actual visits of mass media people to find out "what the Church thinks about it". In his article Fr. Deussen is trying to give an analysis of the pontifical issues up till now. The structures of the communication ethics of Pope Paul VI, he concludes, are individualistic, static, based on natural law and intentional. For the Pope the most important task of social communication is to get the information necessary for the welfare of the individual and single groups for a community. From this there can be derived information ethics for whom in the natural law the basic right is given (active) to have free access to information and (passive) the right to be informed.

\section{RESUMEN}

El Papa Pablo VI no ha desarrollado en ninguno de sus discursos y alocuciones una ética sistemática de los medios de comunicación social. Las entrevistas concretas con los representantes de dichos medios le sirven de ocasión para "reflexionar sobre lo que la Iglesia piensa al respecto". En esta colaboración, Deussen nos presenta un análisis de las declaraciones habídas hasta el presente sobre el periodismo. Llega a la conclusión que las estructuras de la ética de las comunicaciones de Pablo VI son individualistas, estáticas, de derecho natural e intencionales. El fin principal de los medios de comunicación social, según el Papa, es la información para la formación de una sociedad que busque el bien del indivíduo y de los síngulos grupos. De aquí hay que derivar una ética de la información cuya categoría fundamental de derecho natural es el derecho a información, el cual, a su vez, viene definido como derecho activo a acceder a la información y como derecho pasivo sobre la misma. 